\title{
Clinical characteristics and prognostic factors for advanced hepatocellular carcinoma with extrahepatic metastasis
}

\author{
HAJIME AINO $^{1}$, SHUJI SUMIE ${ }^{1}$, TAKASHI NIIZEKI ${ }^{1}$, RYOKO KUROMATSU $^{1}$, NOBUYOSHI TAJIRI ${ }^{1}$, \\ MASAHITO NAKANO ${ }^{1}$, MANABU SATANI $^{1}$, SHINGO YAMADA $^{1}$, SHUSUKE OKAMURA $^{1}$, \\ SHIGEO SHIMOSE $^{1}$, HIROAKI SUMIE ${ }^{1}$, TAKUJI TORIMURA ${ }^{2}$ and MICHIO SATA ${ }^{1}$ \\ ${ }^{1}$ Division of Gastroenterology, Department of Medicine; ${ }^{2}$ Liver Cancer Research Division, \\ Research Center for Innovative Cancer Therapy, Kurume University School of Medicine, Kurume, Fukuoka 830-0011, Japan
}

Received October 8, 2013; Accepted November 27, 2013

DOI: $10.3892 / \mathrm{mco} .2014 .259$

\begin{abstract}
Hepatocellular carcinoma (HCC) is one of the most common malignancies worldwide. The aim of this study was to evaluate whether there are differences in the clinical characteristics and survival between patients with advanced HCC with extrahepatic metastasis who received and those who did not receive previous treatment. Between April, 1998 and April, 2012, a total of 419 HCC patients with extrahepatic metastasis (81 previously untreated and 338 previously treated) were enrolled in this study. The differences in the clinical characteristics, including metastatic sites, were compared between the two groups. In addition, the prognostic predictors among all the patients and among the 81 previously untreated patients were analyzed. The distribution of the major metastatic sites was similar in the two groups; the most frequent site of extrahepatic metastasis was the lungs, followed by the bones, lymph nodes and adrenal glands. The median survival time (MST) among the 419 patients was 6.8 months. The 1-, 2-, 3- and 5-year survival rates were 31.6, 15.3, 9.5 and $2.3 \%$, respectively. No significant differences in survival were observed between patients who received and those who did not receive previous treatment. The multivariate analysis revealed that the Child-Pugh classification, white blood cell count, neutrophil-lymphocyte ratio (NLR) and primary tumor stage were independent predictors of survival for all the patients and for the 81 previously untreated patients. Differences in the clinical characteristics of patients with advanced HCC with extrahepatic metastasis were identified between patients who received and those who did not receive previous treatment. Furthermore, intrahepatic tumor status, Child-Pugh classification, white blood cell count and NLR were demonstrated to
\end{abstract}

Correspondence to: Dr Hajime Aino, Division of Gastroenterology, Department of Medicine, Kurume University School of Medicine, 67 Asahi-machi, Kurume, Fukuoka 830-0011, Japan

E-mail: aino0217@kph.biglobe.ne.jp

Key words: hepatocellular carcinoma, extrahepatic metastasis, survival, previous treatment be independent predictors of survival in HCC patients with extrahepatic metastasis.

\section{Introduction}

Hepatocellular carcinoma (HCC) is one of the most common malignancies worldwide. Recent advances in imaging technology and implementation of surveillance programs for high-risk patients have led to increased detection of early-stage HCC, making curative therapies possible in some patients $(1,2)$. However, the long-term survival of HCC patients remains unsatisfactory, due to the high frequency of intra- and extrahepatic recurrence $(3,4)$. In particular, the development of advanced HCC with extrahepatic metastasis hinders the use of curative therapies, such as surgical resection or radiofrequency ablation, therefore contributing to poor survival. Prior to the approval of sorafenib, several systemic chemotherapeutic regimens had been evaluated for patients with advanced HCC, although no effective therapeutic protocols were identified $(5,6)$. Two randomized placebo-controlled trials demonstrated a survival benefit associated with sorafenib for patients with advanced HCC, including those with extrahepatic metastasis $(7,8)$. As a result, sorafenib is currently considered to be the standard treatment for advanced HCC in the United States, Europe, Japan and a number of other countries $(9,10)$. However, although sorafenib appears to prolong survival, this benefit remains unsatisfactory. Therefore, the development of novel agents and/or combinations is required for patients with advanced HCC with extrahepatic metastasis. To design optimal therapies, it is crucial to understand the clinical characteristics and prognostic factors of these patients.

In our clinic, we encountered several HCC patients whose disease progressed to extrahepatic metastasis, despite the administration of appropriate, repeated treatment for intrahepatic tumors, whereas we also encountered patients who were initially diagnosed with HCC with extrahepatic metastasis at presentation and, therefore, have not been previously treated for HCC. As regards patients with advanced HCC with extrahepatic metastasis, several previous studies only reported the clinicopathological characteristics and prognosis of patients with HCC recurrence or a combined set of previously treated and untreated patients (11-14). We hypothesized 
that the previous treatment of intrahepatic tumors may affect the subsequent pattern of metastasis and prognosis among patients with advanced HCC with extrahepatic metastasis. Therefore, it is crucial to determine the characteristics of pure advanced HCC with extrahepatic metastasis that has not been previously treated. In addition, to the best of our knowledge, no studies have compared the differences between patients with advanced HCC with extrahepatic metastasis who received and those who did not receive previous treatment. Consequently, in this study, we aimed to compare the clinical characteristics and prognostic factors of previously treated and untreated patients with advanced HCC with extrahepatic metastasis.

\section{Patients and methods}

Patients. Between April, 1998 and April, 2012, a total of 419 patients who were diagnosed with advanced HCC with extrahepatic metastasis at the Kurume University School of Medicine, Kurume, Japan, were enrolled in this study. Hepatic functional reserve was determined using the Child-Pugh classification system. HCC tumor staging was performed using the 6th edition of the American Joint Committee on Cancer/Union for International Cancer Control TNM classification system (15). At diagnosis of HCC with extrahepatic metastasis, 338 patients $(80.7 \%)$ had received previous treatment and 81 patients $(19.3 \%)$ were untreated. Previous treatments for intrahepatic tumors included hepatic resection in 65 patients $(19.2 \%)$, percutaneous ethanol injection in 82 patients $(24.3 \%)$, radiofrequency ablation in 83 patients $(24.6 \%)$, transcatheter chemoembolization (TACE) in 204 patients $(60.4 \%)$ and hepatic arterial infusion chemotherapy (HAIC) in 197 patients $(58.3 \%)$. The patients included 353 men $(84.3 \%)$ and 66 women, with a median age of 66.0 years (range, 15-92 years). Overall, 291 patients (69.5\%) were found to be positive for hepatitis C virus (HCV) and 75 patients $(17.9 \%)$ were positive for hepatitis $\mathrm{B}$ virus (HBV) infection. A total of 208, 149 and 62 patients were classified as Child-Pugh class A, B and C, respectively, whereas 55, 186 and 175 patients had T0-2, T3 and T4 stage primary tumors, respectively. Extrahepatic metastases were detected in the lungs in 225 (53.7\%), bones in 165 (39.4\%), lymph nodes in $91(21.7 \%)$ and adrenal glands in 44 patients (10.5\%).

Diagnosis of HCC and evaluation of extrahepatic lesions. The diagnosis of HCC was radiologically confirmed by hyperintensity in the arterial phase and washout in the venous and delayed phase, using either contrast-enhanced computed tomography (CT) or magnetic resonance imaging (MRI) (9) and/or by elevated serum levels of $\alpha$-fetoprotein (AFP) and des- $\gamma$-carboxy prothrombin (DCP). Tumor biopsy was performed in cases in which imaging findings were not consistent with the characteristic features of HCC, or when tumor marker levels were not elevated. To evaluate extrahepatic metastasis, pulmonary lesions were detected on chest $\mathrm{X}$-ray or chest CT, which were routinely performed at the first visit or every 3-6 months during the follow-up period. Additional examinations, such as bone scintigraphy and brain CT or MRI, were indicated upon development of symptoms attributable to extrahepatic metastasis. These examinations were also conducted when AFP and/or DCP levels were elevated and the elevation(s) could not be attributed to the status of the intrahepatic lesion(s). Positron emission tomography/CT studies were performed as a supplemental examination.

Follow-up and endpoint. Following HCC diagnosis, each patient was carefully followed up with respect to intrahepatic lesions and extrahepatic metastases. Serum biochemistries, AFP and DCP levels were measured and ultrasonography was performed every 1-2 months. Contrast CT or MRI was performed every 2-6 months. Other imaging modalities were used as necessary. The endpoint of this study was the date of death, or last follow-up visit; the closing date was August, 2012. The median duration of the follow-up was 5.8 months (range, 0.2-111.9 months).

Statistical analysis. The continuous variables are expressed as median values (range). A comparison analysis between patients who received and those who did not receive previous treatment was performed using the Chi-square test for discrete variables and the Mann-Whitney $U$ test for continuous variables. Overall survival was determined by the Kaplan-Meier analysis and the differences between subgroups were compared with log-rank tests. A Cox proportional hazards stepwise model was used for univariate and multivariate analysis, in order to identify any independent variables associated with overall survival. Data from these models are expressed as hazard ratios (HRs) and 95\% confidence intervals (CIs). All P values were two-tailed and $\mathrm{P}<0.05$ was considered to indicate a statistically significant difference. The statistical analysis was performed using SPSS software, version 20 (SPSS, Inc., Chicago, IL, USA).

\section{Results}

Clinical characteristics of patients. A comparison of the clinical characteristics of patients who received and those who did not receive previous treatment is shown in Table I. Previously treated patients were significantly more likely to have $\mathrm{HCV}$ infection, Child-Pugh class $\mathrm{B}+\mathrm{C}$ and a low primary tumor stage, compared to previously untreated patients. Previously untreated patients exhibited a significantly lower neutrophil-lymphocyte ratio (NLR) and higher aspartate aminotransferase (AST) levels, white blood cell counts, platelet counts and DCP levels, compared to previously treated patients. The major metastatic sites, which were the lungs, bones, lymph nodes and adrenal glands were similar in the two groups.

Survival and predictive factors in all the patients. The cumulative survival curve of the 419 patients is shown in Fig. 1. The median survival time (MST) for these patients was 6.8 months. The 1-, 2-, 3- and 5-year survival rates were 31.6, 15.3, 9.5 and $2.3 \%$, respectively. A Cox proportional hazards regression analysis was performed to identify independent predictors of survival (Table II). The results of the univariate analysis demonstrated that Child-Pugh class $(\mathrm{B}+\mathrm{C})$, white blood cell count $\left(\geq 6.0 \times 10^{9} / \mathrm{l}\right)$, NLR $(\geq 4.0)$, AFP levels $(\geq 200 \mathrm{ng} / \mathrm{ml})$ and 
Table I. Comparison of clinical characteristics between previously treated and untreated patients.

\begin{tabular}{|c|c|c|c|}
\hline Characteristics & Previously untreated patients $(\mathrm{n}=81$ ) & Previously treated patients $(n=338)$ & P-value \\
\hline Gender (male/female) & $70 / 11$ & $283 / 55$ & 0.523 \\
\hline Age, years (range) & $63(15-80)$ & $67(30-92)$ & $<0.001$ \\
\hline Etiology (HCV/HBV/other) & $44 / 18 / 19$ & $246 / 57 / 35$ & 0.006 \\
\hline Child-Pugh class (A/B/C) & $56 / 17 / 8$ & $152 / 132 / 54$ & $<0.001$ \\
\hline $\operatorname{AST}(\mathrm{U} / \mathrm{l})$ & $52(12-280)$ & $36(6-412)$ & $<0.001$ \\
\hline White blood cell count (x109/1) & $5.7(2.8-20.3)$ & $4.4(1.4-20.5)$ & $<0.001$ \\
\hline NLR & $2.8(0.8-18.0)$ & $3.1(0.6-46.5)$ & 0.038 \\
\hline Platelet count $\left(\times 10^{9} / \mathrm{l}\right)$ & $139(33-675)$ & $101(20-970)$ & $<0.001$ \\
\hline $\mathrm{AFP}(\mathrm{ng} / \mathrm{ml})$ & $1444.9(2.2-3,311,794.0)$ & $524.9(1.5-1,904,794.0)$ & 0.177 \\
\hline $\mathrm{DCP}(\mathrm{mAU} / \mathrm{ml})$ & $11400(19-75,000)$ & $887.5(8-75,000)$ & $<0.001$ \\
\hline Primary tumor stage ${ }^{\mathrm{a}}(\mathrm{T} 0-2 / \mathrm{T} 3 / \mathrm{T} 4)$ & $2 / 30 / 49$ & $53 / 156 / 126^{b}$ & $<0.001$ \\
\hline \multicolumn{4}{|l|}{ Site of extrahepatic metastasis } \\
\hline Lungs & $53.1 \%(43)$ & $53.8 \%(182)$ & 0.905 \\
\hline Bones & $43.2 \%(35)$ & $38.5 \%(130)$ & 0.410 \\
\hline Lymph nodes & $29.6 \%(24)$ & $19.8 \%(67)$ & 0.059 \\
\hline Adrenal glands & $13.6 \%(11)$ & $9.8 \%(33)$ & 0.306 \\
\hline Peritoneum and pleura & $2.5 \%(2)$ & $7.4 \%(25)$ & 0.107 \\
\hline Diaphragm & $4.9 \%(4)$ & $5.0 \%(17)$ & 0.982 \\
\hline Brain & $1.2 \%(1)$ & $3.0 \%(10)$ & 0.387 \\
\hline
\end{tabular}

${ }^{a}$ TNM classification. Continuous variables are presented as median (range). HCV, hepatitis C virus; HBV, hepatitis B virus; AST, aspartate aminotransferase; NLR, neutrophil-lymphocyte ratio; AFP, $\alpha$-fetoprotein; DCP, des- $\gamma$-carboxy prothrombin. ${ }^{a}$ TNM classification, ${ }^{b}$ Except in the cases of unclassified $\mathrm{T}$ factor (3/338).

Table II. Univariate and multivariate analysis of survival in all 419 patients with HCC and extrahepatic metastasis.

\begin{tabular}{|c|c|c|c|c|}
\hline Variables & Univariate HR (95\% CI) & P-value & Multivariate HR (95\% CI) & P-value \\
\hline Gender (male) & $0.96(0.71-1.29)$ & 0.773 & - & - \\
\hline Age $(\geq 65$ years $)$ & $1.10(0.89-1.37)$ & 0.374 & - & - \\
\hline Etiology (HCV infection) & $1.01(0.80-1.28)$ & 0.909 & - & - \\
\hline Child-Pugh class $(\mathrm{B}+\mathrm{C})$ & $2.82(2.25-3.53)$ & $<0.001$ & $2.80(2.23-3.52)$ & $<0.001$ \\
\hline $\operatorname{AST}(\geq 80 \mathrm{U} / \mathrm{l})$ & $1.15(0.87-1.51)$ & 0.335 & - & - \\
\hline White blood cell count $\left(\geq 6.0 \times 10^{9} / 1\right)$ & $1.85(1.45-2.35)$ & $<0.001$ & $1.82(1.43-2.33)$ & $<0.001$ \\
\hline $\operatorname{NLR}(\geq 4.0)$ & $2.48(1.96-3.13)$ & $<0.001$ & $1.89(1.48-2.41)$ & $<0.001$ \\
\hline Platelet count $\left(\geq 120 \times 10^{9} / 1\right)$ & $0.93(0.75-1.15)$ & 0.504 & - & - \\
\hline $\operatorname{AFP}(\geq 200$ ng/ml $)$ & $1.74(1.39-2.18)$ & $<0.001$ & $1.48(1.18-1.87)$ & 0.001 \\
\hline $\mathrm{DCP}(\geq 200 \mathrm{mAU} / \mathrm{ml})$ & $1.10(0.99-1.22)$ & 0.073 & - & - \\
\hline Primary tumor stage ${ }^{a}(\mathrm{~T} 4)$ & $1.57(1.27-1.95)$ & $<0.001$ & $1.43(1.14-1.79)$ & 0.002 \\
\hline Previous treatment (present) & $1.13(0.87-1.48)$ & 0.370 & - & - \\
\hline \multicolumn{5}{|l|}{ Site of extrahepatic metastasis } \\
\hline Lungs & $0.93(0.75-1.15)$ & 0.480 & - & - \\
\hline Bones & $1.23(0.99-1.52)$ & 0.066 & - & - \\
\hline Lymph nodes & $0.96(0.74-1.24)$ & 0.732 & - & - \\
\hline
\end{tabular}

aTNM classification. HCC, hepatocellular carcinoma; HR, hazard ratio; CI, confidence interval; HCV, hepatitis C virus; AST, aspartate aminotransferase;

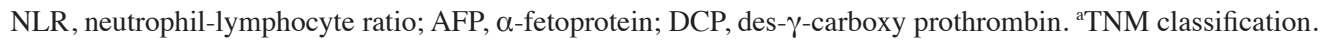

primary tumor stage (T4) were significant risk factors that adversely affected survival. The multivariate analysis identified Child-Pugh class $\mathrm{B}+\mathrm{C}(\mathrm{HR}=2.80 ; 95 \% \mathrm{CI}: 2.23-3.52 ; \mathrm{P}<0.001)$, white blood cell count $\geq 6.0 \times 10^{9} / 1$ (HR=1.82; 95\% CI: $1.43-2.33$; $\mathrm{P}<0.001), \mathrm{NLR} \geq 4.0$ (HR=1.89; 95\% CI: 1.48-2.41; $\mathrm{P}<0.001$ ), AFP $\geq 200 \mathrm{ng} / \mathrm{ml}(\mathrm{HR}=1.48 ; 95 \% \mathrm{CI}: 1.18-1.87 ; \mathrm{P}=0.001)$ and primary tumor stage $\mathrm{T} 4(\mathrm{HR}=1.43 ; 95 \% \mathrm{CI}$ : $1.14-1.79 ; \mathrm{P}=0.002)$ as independent predictors of survival.

Survival and predictive factors in 81 previously untreated patients. The cumulative survival curve of the 81 patients who did not receive previous treatment is shown in Fig. 2. 
Table III. Univariate and multivariate analysis of survival in previously untreated patients with HCC and extrahepatic metastasis.

\begin{tabular}{|c|c|c|c|c|}
\hline Variables & Univariate HR (95\% CI) & P-value & Multivariate HR (95\% CI) & P-value \\
\hline Gender (male) & $1.33(0.66-2.72)$ & 0.427 & - & - \\
\hline Age ( $\geq 65$ years) & $1.04(0.64-1.68)$ & 0.872 & - & - \\
\hline Etiology (HCV infection) & $0.79(0.49-1.29)$ & 0.351 & - & - \\
\hline Child-Pugh class $(\mathrm{B}+\mathrm{C})$ & $5.66(3.22-9.94)$ & $<0.001$ & $6.03(3.31-10.99)$ & $<0.001$ \\
\hline $\operatorname{AST}(\geq 80 \mathrm{U} / 1)$ & $1.13(0.66-1.93)$ & 0.659 & - & - \\
\hline White blood cell count $\left(\geq 6.0 \times 10^{9} / 1\right)$ & $1.97(1.18-3.29)$ & 0.009 & $1.85(1.09-3.15)$ & 0.023 \\
\hline $\operatorname{NLR}(\geq 4.0)$ & $2.57(1.44-4.56)$ & 0.001 & $1.86(1.01-3.43)$ & 0.047 \\
\hline Platelet count $\left(\geq 120 \times 10^{9} / 1\right)$ & $1.25(0.75-2.08)$ & 0.387 & - & - \\
\hline $\operatorname{AFP}(\geq 200$ ng/ml $)$ & $1.43(0.85-2.39)$ & 0.175 & - & - \\
\hline $\mathrm{DCP}(\geq 200 \mathrm{mAU} / \mathrm{ml})$ & $1.05(0.56-1.96)$ & 0.885 & - & - \\
\hline Primary tumor stage ${ }^{a}(\mathrm{~T} 4)$ & $1.64(1.00-2.67)$ & 0.048 & $1.82(1.10-2.99)$ & 0.019 \\
\hline \multicolumn{5}{|l|}{ Site of extrahepatic metastasis } \\
\hline Lungs & $0.93(0.57-1.53)$ & 0.783 & - & - \\
\hline Bones & $1.30(0.800-2.13)$ & 0.292 & - & - \\
\hline Lymph nodes & $1.23(0.69-2.19)$ & 0.482 & - & - \\
\hline
\end{tabular}

${ }^{a}$ TNM classification. HCC, hepatocellular carcinoma; HR, hazard ratio; CI, confidence interval; HCV, hepatitis C virus; AST, aspartate aminotransferase; NLR, neutrophil-lymphocyte ratio; AFP, $\alpha$-fetoprotein; DCP, des- $\gamma$-carboxy prothrombin. ${ }^{\text {aTNM classification. }}$

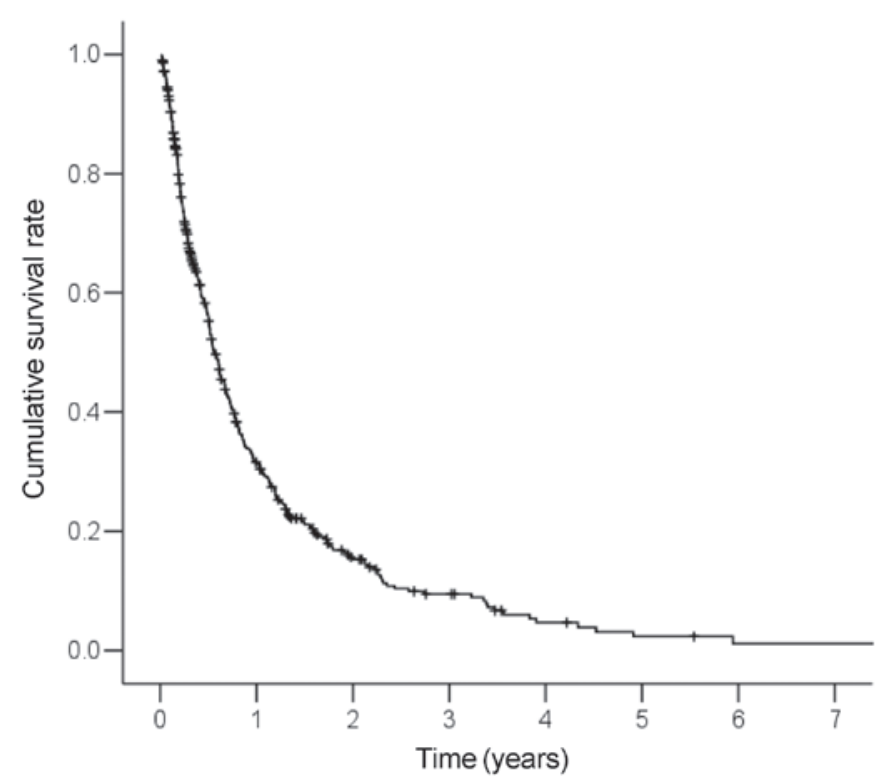

Figure 1. Cumulative survival curve for the 419 patients. The median survival time of all patients was 6.8 months. The 1-, 2-, 3- and 5-year survival rates were $31.6,15.3,9.5$ and $2.3 \%$, respectively.

The MST for these patients was 7.4 months. The 1-, 2-, 3- and 5-year survival rates were 33.7, 18.8, 11.9 and 2.3\%, respectively. No significant differences in survival between patients who received and those who did not receive previous treatment were observed $(\mathrm{P}=0.369)$. A Cox proportional hazards regression analysis was performed to identify independent predictors of survival (Table III). The results of the univariate analysis revealed that Child-Pugh class $(\mathrm{B}+\mathrm{C})$, white blood cell count $\left(\geq 6.0 \times 10^{9} / 1\right)$, NLR $(\geq 4.0)$ and primary tumor stage (T4) were significant risk factors that adversely affected survival. The multivariate analysis identified Child-Pugh class $\mathrm{B}+\mathrm{C}(\mathrm{HR}=6.03$; 95\% CI: 3.31-10.99; $\mathrm{P}<0.001)$, white blood

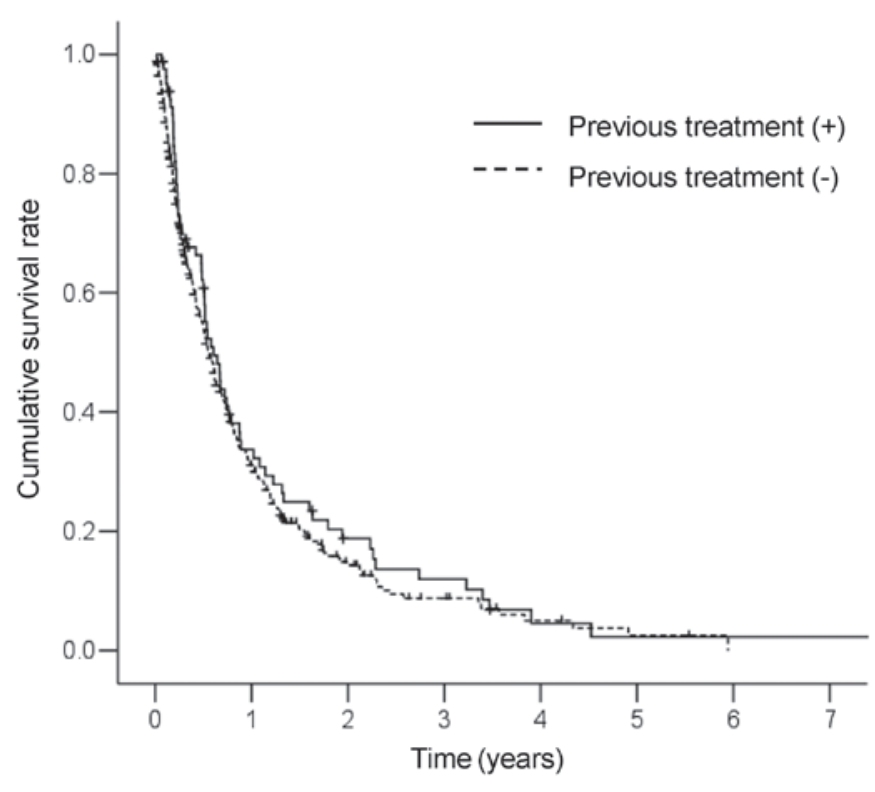

Figure 2. Comparison of cumulative survival curves between patients who received and those who did not receive previous treatment. The median survival times of previously treated and untreated patients were 6.7 and 7.4 months, respectively $(\mathrm{P}=0.369)$.

cell count $\geq 6.0 \times 10^{9} / 1(\mathrm{HR}=1.85 ; 95 \% \mathrm{CI}: 1.09-3.15 ; \mathrm{P}=0.023)$, $\mathrm{NLR} \geq 4.0(\mathrm{HR}=1.86 ; 95 \% \mathrm{CI}: 1.01-3.43 ; \mathrm{P}=0.047)$ and primary tumor stage T4 $(\mathrm{HR}=1.82 ; 95 \% \mathrm{CI}: 1.10-2.99 ; \mathrm{P}=0.019)$ as independent predictors of survival.

\section{Discussion}

It has not been determined whether previous treatment affects the clinical characteristics and prognosis in patients with advanced HCC and extrahepatic metastasis. In this study, we aimed to evaluate whether there are differences between 
patients with advanced HCC and extrahepatic metastasis who received and those who did not receive previous treatment. Our results revealed differences in various clinical characteristics between these two groups of patients. Previously treated patients were more likely to exhibit low white blood cell counts, low platelet counts and poor liver function compared to previously untreated patients. A possible explanation for this observation is that leukocytopenia and thrombocytopenia may have been caused by previously administered anticancer drugs, as several patients had repeatedly undergone TACE and HAIC for intrahepatic tumors. In addition, repeated cancer recurrence and various treatments for intrahepatic tumors may have contributed to decreased liver function. Of note, previously untreated patients had more advanced-stage intrahepatic tumors compared to previously treated patients. Previous studies suggested that residual HCC following various treatments is associated with increased malignant potential compared to untreated HCC (16-18). Therefore, we suggest that aggressive treatment may result in earlier extrahepatic metastasis, despite a less advanced intrahepatic tumor stage, in previously treated patients. In this study, no significant differences in survival between the two groups were observed, although the groups differed in liver function and intrahepatic tumor stage. A possible explanation for this lack of difference is that worse liver function and an increased malignant potential of previously treated patients may offset the more advanced stage of intrahepatic tumors of previously untreated patients.

The major sites of HCC metastasis included the lungs, lymph nodes, bones and adrenal glands (13,14,19-21). Yoo et al (20) reported that the most frequent metastatic sites in 251 previously untreated HCC patients with extrahepatic metastasis were the lungs $(67.3 \%)$, lymph nodes (37.5\%), bones (18.3\%) and adrenal glands (7.6\%). Jun et al (14) indicated that in HCC patients with extrahepatic recurrence following hepatic resection, frequent metastatic sites included the lungs (41.9\%), lymph nodes $(19.9 \%)$ and bones $(13.2 \%)$. In the present study, the most frequent site of extrahepatic metastasis was the lungs, followed by the bones, lymph nodes and adrenal glands, regardless of previous treatment of intrahepatic tumors. These results are similar to those previously reported and suggest that previous treatment does not affect the metastatic pattern of HCC.

Several studies reported that in HCC patients with extrahepatic metastasis, intrahepatic tumor status and Child-Pugh classification are independent prognostic factors $(12,13,20)$. Similarly, in the present study, the multivariate analysis demonstrated that these factors were independent predictors of survival in HCC patients with extrahepatic metastasis, regardless of previous treatment. Sorafenib is an oral systemic agent that prolongs overall survival and has become the standard treatment for patients with advanced HCC, including those with extrahepatic metastasis. However, even the survival rates achieved with sorafenib remain unsatisfactory. Several studies reported that the characteristics of primary tumor progression, such as vascular invasion, tumor size and tumor number, are independent risk factors for extrahepatic metastasis following curative resection $(14,22)$. Thus, the control of intrahepatic tumors may be important for the prevention of further extrahepatic metastasis. Pinter et al (23) reported that the MST of TACE alone (9.2 months) was similar to that of sorafenib alone (7.4 months) in patients with advanced HCC, including those with extrahepatic metastasis. Jun et al (14) reported that in $240 \mathrm{HCC}$ patients with extrahepatic metastasis, the control of intrahepatic tumors was a favorable prognostic factor for survival: the MST of patients exhibiting a treatment response was significantly longer compared to that of patients who did not respond to treatment (521 vs. 170 days; $\mathrm{P}<0.001)$. Consequently, in advanced HCC patients with extrahepatic metastasis, a combination of intrahepatic local treatments and sorafenib may be useful, as the malignant potential of intrahepatic tumors is associated with extrahepatic spread and survival.

Hepatic reserve is important for hepatic resection and metabolism of anticancer drugs, including sorafenib. Pinter et al (24) reported that the risk of high-grade toxicities associated with sorafenib may be increased in patients with advanced liver dysfunction. Our previous study of advanced HCC patients treated with HAIC demonstrated that liver dysfunction necessitating treatment suspension or discontinuation occurred more frequently in patients with Child-Pugh class B compared to patients with Child-Pugh class A disease (25). Such insufficient treatment may lead to further liver dysfunction, due to intrahepatic tumor progression, resulting in poor survival. Therefore, we considered liver function to be an important predictor of survival.

In this study, we also demonstrated that an elevated white blood cell count associated with a high NLR was a significant independent predictor of survival in HCC patients with extrahepatic metastasis. Recently, various markers of systemic inflammatory responses, including cytokines, C-reactive protein and absolute blood neutrophil or lymphocyte count, as well as their ratio (including NLR), have been investigated for their prognostic roles in cancer. Of these, NLR is one of the most simple and effective markers of inflammation and is linked with poor prognosis in various cancer types (26-28). Several studies demonstrated that an elevated NLR was associated with worse survival in patients with HCC who underwent radiofrequency ablation, TACE, resection and liver transplantation (29-32). However, the exact association between a high NLR and poor prognosis has not been fully elucidated. One possible explanation is that patients with an elevated NLR have relative lymphocytopenia, leading to a weaker lymphocyte-mediated immune response to the tumor due to a decreasing T4/T8 ratio (33). As a result, these patients may experience a more rapid tumor progression and, therefore, have a poor prognosis. Another explanation is that the increased neutrophil numbers may modify and provide an adequate environment for tumor progression and development. Neutrophils have been shown to promote tumor growth and metastasis by secreting chemokines, vascular endothelial growth factor and matrix metalloproteinase-9, which are involved in the angiogenesis that promotes tumor development (34-36). Thus, a high neutrophil level may offer a growth advantage for HCC through the increase of these pro-angiogenic factors, resulting in increased extrahepatic metastasis and worse survival in HCC patients.

In conclusion, we demonstrated that there are differences in the clinical characteristics and no significant differences in survival between patients with advanced HCC with extrahepatic metastasis who received and those who did not receive previous treatment. Moreover, we demonstrated that 
intrahepatic tumor status, Child-Pugh classification, white blood cell count and NLR are independent predictors of survival in HCC patients with extrahepatic metastasis, regardless of previous treatment of intrahepatic tumors.

\section{References}

1. Takayama T, Makuuchi M, Hirohashi S, et al: Early hepatocellular carcinoma as an entity with a high rate of surgical cure. Hepatology 28: 1241-1246, 1998.

2. Zhang BH, Yang BH and Tang ZY: Randomized controlled trial of screening for hepatocellular carcinoma. J Cancer Res Clin Oncol 130: 417-422, 2004.

3. Portolani N, Coniglio A, Ghidoni S, et al: Early and late recurrence after liver resection for hepatocellular carcinoma: prognostic and therapeutic implications. Ann Surg 243: 229-235, 2006.

4. Yang Y, Nagano H, Ota H, et al: Patterns and clinicopathologic features of extrahepatic recurrence of hepatocellular carcinoma after curative resection. Surgery 141: 196-202, 2007.

5. Burroughs A, Hochhauser D and Meyer T: Systemic treatment and liver transplantation for hepatocellular carcinoma: two ends of the therapeutic spectrum. Lancet Oncol 5: 409-418, 2004.

6. Nowak AK, Chow PK and Findlay M: Systemic therapy for advanced hepatocellular carcinoma: a review. Eur J Cancer 40 1474-1484, 2004.

7. Llovet JM, Ricci S, Mazzaferro V, et al: Sorafenib in advanced hepatocellular carcinoma. N Engl J Med 359: 378-390, 2008

8. Cheng AL, Kang YK, Chen Z, et al: Efficacy and safety of sorafenib in patients in the Asia-Pacific region with advanced hepatocellular carcinoma: a phase III randomised, double-blind, placebo-controlled trial. Lancet Oncol 10: 25-34, 2009.

9. Bruix J and Sherman M; American Association for the Study of Liver Diseases: Management of hepatocellular carcinoma: an update. Hepatology 53: 1020-1022, 2011.

10. Kudo M, Izumi N, Kokudo N, et al; HCC Expert Panel of Japan Society of Hepatology: Management of hepatocellular carcinoma in Japan: Consensus-Based Clinical Practice Guidelines proposed by the Japan Society of Hepatology (JSH) 2010 updated version. Dig Dis 29: 339-364, 2011.

11. Sasaki A, Kai S, Endo Y, et al: Hepatitis B virus infection predicts extrahepatic metastasis after hepatic resection in patients with large hepatocellular carcinoma. Ann Surg Oncol 14: 3181-3187, 2007.

12. Uka K, Aikata H, Takaki S, et al: Clinical features and prognosis of patients with extrahepatic metastases from hepatocellular carcinoma. World J Gastroenterol 13: 414-420, 2007.

13. Uchino K, Tateishi R, Shiina S, et al: Hepatocellular carcinoma with extrahepatic metastasis: clinical features and prognostic factors. Cancer 117: 4475-4483, 2011.

14. Jun L, Zhenlin Y, Renyan G, et al: Independent factors and predictive score for extrahepatic metastasis of hepatocellular carcinoma following curative hepatectomy. Oncologist 17: 963-969, 2012.

15. Pawlik TM, Esnaola NF and Vauthey JN: Surgical treatment of hepatocellular carcinoma: similar long-term results despite geographic variations. Liver Transpl 10 (Suppl 1): S74-S80, 2004.

16. Shim JH, Park JW, Kim JH, et al: Association between increment of serum VEGF level and prognosis after transcatheter arterial chemoembolization in hepatocellular carcinoma patients. Cancer Sci 99: 2037-2044, 2008.

17. Paez-Ribes M, Allen E, Hudock J, et al: Antiangiogenic therapy elicits malignant progression of tumors to increased local invasion and distant metastasis. Cancer Cell 15: 220-231, 2009.

18. Kong J, Kong J, Pan B, et al: Insufficient radiofrequency ablation promotes angiogenesis of residual hepatocellular carcinoma via HIF-1alpha/VEGFA. PLoS One 7: e37266, 2012.
19. Katyal S, Oliver JH III, Peterson MS, Ferris JV, Carr BS and Baron RL: Extrahepatic metastases of hepatocellular carcinoma. Radiology 216: 698-703, 2000

20. Yoo DJ, Kim KM, Jin YJ, et al: Clinical outcome of 251 patients with extrahepatic metastasis at initial diagnosis of hepatocellular carcinoma: does transarterial chemoembolization improve survival in these patients? J Gastroenterol Hepatol 26: 145-154, 2011.

21. Natsuizaka M, Omura T, Akaike T, et al: Clinical features of hepatocellular carcinoma with extrahepatic metastases. J Gastroenterol Hepatol 20: 1781-1787, 2005.

22. Ochiai T, Ikoma H, Okamoto K, Kokuba Y, Sonoyama T and Otsuji E: Clinicopathologic features and risk factors for extrahepatic recurrences of hepatocellular carcinoma after curative resection. World J Surg 36: 136-143, 2012.

23. Pinter M, Hucke F, Graziadei I, et al: Advanced-stage hepatocellular carcinoma: transarterial chemoembolization versus sorafenib. Radiology 263: 590-599, 2012.

24. Pinter M, Sieghart W, Graziadei I, et al: Sorafenib in unresectable hepatocellular carcinoma from mild to advanced stage liver cirrhosis. Oncologist 14: 70-76, 2009.

25. Niizeki T, Sumie S, Torimura T, et al: Serum vascular endothelial growth factor as a predictor of response and survival in patients with advanced hepatocellular carcinoma undergoing hepatic arterial infusion chemotherapy. J Gastroenterol 47: 686-695, 2012.

26. Ding PR, An X, Zhang RX, et al: Elevated preoperative neutrophil to lymphocyte ratio predicts risk of recurrence following curative resection for stage IIA colon cancer. Int J Colorectal Dis 25: 1427-1433, 2010.

27. Jung MR, Park YK, Jeong O, et al: Elevated preoperative neutrophil to lymphocyte ratio predicts poor survival following resection in late stage gastric cancer. J Surg Oncol 104: 504-510, 2011.

28. Stotz M, Gerger A, Eisner F, et al: Increased neutrophillymphocyte ratio is a poor prognostic factor in patients with primary operable and inoperable pancreatic cancer. Br J Cancer 109: 416-421, 2013.

29. Gomez D, Farid S, Malik HZ, et al: Preoperative neutrophil-tolymphocyte ratio as a prognostic predictor after curative resection for hepatocellular carcinoma. World J Surg 32: 1757-1762, 2008.

30. Halazun KJ, Hardy MA, Rana AA, et al: Negative impact of neutrophil-lymphocyte ratio on outcome after liver transplantation for hepatocellular carcinoma. Ann Surg 250: 141-151, 2009.

31. Huang ZL, Luo J, Chen MS, Li JQ and Shi M: Blood neutrophil-to-lymphocyte ratio predicts survival in patients with unresectable hepatocellular carcinoma undergoing transarterial chemoembolization. J Vasc Interv Radiol 22: 702-709, 2011.

32. Chen TM, Lin CC, Huang PT and Wen CF: Neutrophil-tolymphocyte ratio associated with mortality in early hepatocellular carcinoma patients after radiofrequency ablation. J Gastroenterol Hepatol 27: 553-561, 2012.

33. Chew V, Tow C, Teo M, et al: Inflammatory tumour microenvironment is associated with superior survival in hepatocellular carcinoma patients. J Hepatol 52: 370-379, 2010.

34. Coussens LM, Tinkle CL, Hanahan D and Werb Z: MMP-9 supplied by bone marrow-derived cells contributes to skin carcinogenesis. Cell 103: 481-490, 2000.

35. Strieter RM, Burdick MD, Mestas J, Gomperts B, Keane MP and Belperio JA: Cancer CXC chemokine networks and tumour angiogenesis. Eur J Cancer 42: 768-778, 2006.

36. Gong Y and Koh DR: Neutrophils promote inflammatory angiogenesis via release of preformed VEGF in an in vivo corneal model. Cell Tissue Res 339: 437-448, 2010. 